\title{
Thermodynamic properties and structural stability of thorium dioxide
}

\author{
Yong $\mathrm{Lu},{ }^{1} \mathrm{Yu}$ Yang, ${ }^{1}$ and Ping Zhang ${ }^{1,2, *}$ \\ ${ }^{1}$ LCP, Institute of Applied Physics and Computational Mathematics, Beijing 100088, China \\ ${ }^{2}$ Center for Applied Physics and Technology, Peking University, Beijing 100871, China
}

(Dated: May 17, 2012)

\begin{abstract}
Using density functional theory (DFT) calculations, we have systematically investigated the thermodynamic properties and structural stabilities of thorium dioxide $\left(\mathrm{ThO}_{2}\right)$. Based on the calculated phonon dispersion curves, we calculate the thermal expansion coefficient, bulk modulus, and heat capacities at different temperatures for $\mathrm{ThO}_{2}$ under the quasi-harmonic approximation. All the results are in good agreement with corresponding experiments proving the validity of our methods. Our theoretical studies can help people more clearly understand the thermodynamic behaviors of $\mathrm{ThO}_{2}$ at different temperatures. In addition, we have also studied possible defect formations and diffusion behaviors of helium in $\mathrm{ThO}_{2}$, to discuss its structural stability. It is found that in intrinsic $\mathrm{ThO}_{2}$ without any Fermi energy shifts, the interstitial $\mathrm{Th}_{i}^{4+}$ defect other than oxygen or thorium vacancies, interstitial oxygen, and any kinds of Frenkel pairs, is most probable to form with an energy release of $1.74 \mathrm{eV}$. However, after upshifting the Fermi energy, the formation of the other defects also becomes possible. For helium diffusion, we find that only through the thorium vacancy can it happen with the small energy barrier of $0.52 \mathrm{eV}$. Otherwise, helium atoms can hardly incorporate or diffuse in $\mathrm{ThO}_{2}$. Our results indicate that people should prevent upshifts of the Fermi energy of $\mathrm{ThO}_{2}$ to avoid the formation of thorium vacancies and so as to prevent helium caused damages.
\end{abstract}

PACS numbers: 63.20.dk, 65.40.-b, 61.72.-y, 66.30.J-.

\section{INTRODUCTION}

As the world's demands for energy keep growing, corresponding researches on developing new energy sources or enhancing the energy-consuming efficiency are attracting more and more attentions. Until now, the fissile nuclear reactor is still a very important energy source, in which uranium dioxide $\left(\mathrm{UO}_{2}\right)$ has been the main fuel component for many years [1 $[5]$. However, during the burning cycle of $\mathrm{UO}_{2}$, considerable amounts of redioactive elements emerge in the reaction waste [ $[6]$. And such radioactive waste results in very troublesome long-term storage requirements. Based on these facts, many efforts have been done to look for possible substitutions of $\mathrm{UO}_{2}$. Thorium based materials, which are naturally abundant, are identified as good candidates for replacing $\mathrm{UO}_{2}$ in fissile nuclear reactors because they are able to produce fewer transuranic (TRU) compared to uraniumand plutonium-based fuels. And although ${ }^{233} \mathrm{Th}$ is not fissile, it can absorb a slow neutron and then form fissile ${ }^{233} \mathrm{U}$ just undergoing multiple beta-decays [7]. Moreover, because of the good solubility between thorium dioxide $\left(\mathrm{ThO}_{2}\right)$ and other transuranic dioxides, using the mix oxides (MOX) of thorium and plutonium in nuclear reactors can also help reducing the large plutonium stockpile while maintaining acceptable safety and control characteristics of the reactor system [8]. In comparison with previous uranium-based fuels, the thorium-based fuels also have many additional physical advantages, such as

\footnotetext{
*Author to whom correspondence should be addressed. E-mail: zhang_ping@iapcm.ac.cn
}

higher melting points, higher corrosion resistivity, lower thermal expansion coefficients, and higher thermal conductivity [9]. In recent years, the thorium-based fuels have already been tested in different reactors [7, 10 12].

As in the front position of actinide element, thorium and its compounds have been studied ever since 1950. At the earliest stage, a series of experimental measurements have been carried out on the thermal properties of $\mathrm{ThO}_{2}$ such as the thermal expansion coefficient, heat capacity, and thermal conductivity [13 22]. In 1997, Bakker et al. [23] presented a conclusion and comparison for the measured values. On the other side, Chadwick and Graham [24], Allen and Tucker [25], and Veal et al. [26] investigated the valence-band structures of thorium and its oxides by means of X-ray photoemission spectroscopy. And the pressure-induced phase transition of $\mathrm{ThO}_{2}$ has been studied by Jayaraman [27], Dancausse [28], and Idiri 29] et al. experimently. Recently in 2006, researchers from the international atomic energy agency (IAEA) built a thermal-physical database of materials for light water reactors and heavy water reactors [30]. And the earlier experimental measurements are re-addressed.

Despite the vast experimental measurements, it is to our surprise that no one has ever theoretically investigated the thermodynamic properties and defect behaviors for $\mathrm{ThO}_{2}$, which are critically important for its usage in thermonuclear reactors. Only recently, several theoretical studies have been carried out on the mechanical and electronic properties [31 33], phase transition behaviors [34, 35], and elastic and optical properties 36] for $\mathrm{ThO}_{2}$. Our previous study has already proven that the thorium $5 f$ states is no longer localized after electronic hybridizations, and density functional theory calculations are enough to produce correct descriptions for 
the ground-state properties of $\mathrm{ThO}_{2}$ [35]. So in our present paper, we decide to systematically investigate the thermodynamic properties and structural stabilities of $\mathrm{ThO}_{2}$, by using density functional theory calculations. The thermodynamic stability will be discussed based on the calculated thermal parameters, while the structural stability will be discussed by calculating the formation energy of different kinds of defects, and diffusion energy barriers of helium in $\mathrm{ThO}_{2}$. The rest of the paper is organized as follows. The computation methods are introduced in Section II. The discussions about the thermodynamic properties and structural stabilities of $\mathrm{ThO}_{2}$ are presented in Section III. Finally, we give our conclusions in Section IV.

\section{CALCULATION METHODS}

The density functional theory (DFT) calculations are carried out using the Vienna ab initio simulations package (VASP) [37, 38] with the projector-augmented-wave (PAW) potential methods [39]. The cutoff energy for the plane-wave basis set is set to $500 \mathrm{eV}$. The exchange and correlation effects are described by generalized gradient approximation (GGA) in the Perdew-Burke-Ernzerhof (PBE) form [40]. A $2 \times 2 \times 2$ supercell is employed to study defect formation and helium diffusions inside $\mathrm{ThO}_{2}$. For calculations of the unit cell (12 total atoms) and $2 \times 2 \times 2$ supercell (96 total atoms), the integration over the Brillouin Zone is done on $13 \times 13 \times 13$ and $5 \times 5 \times 5 k$-ponit meshes generated using the Monkhorst-Pack [41] method, which are both proven to be sufficient for energy convergence of less than $1.0 \times 10^{-4} \mathrm{eV}$ per atom. During the supercell calculations, the shape and size of the supercell are fixed while all the ions are free to relax until the forces on them are less than $0.01 \mathrm{eV} / \AA$.

For a semiconductor, the Helmholtz free energy $F$ at volume $V$ and temperature $T$ can be expressed as

$$
F(V, T)=E(V)+F_{v i b}(V, T),
$$

where $E(V)$ stands for the ground-state electronic energy, $F_{v i b}(V, T)$ is the phonon free energy at a given unit cell volume $V$. Within quasi-hamonic approximation (QHA), $F_{v i b}(V, T)$ can be evaluated by

$$
F_{v i b}(V, T)=k_{B} T \sum_{j, \mathbf{q}} \ln \left[2 \sinh \left(\frac{\hbar \omega_{j}(\mathbf{q}, V)}{2 k_{B} T}\right)\right],
$$

where $\omega_{j}(\mathbf{q}, V)$ is the phonon frequency of the $j$ th phonon mode with wave vector $\mathbf{q}$ at fixed $V$, and $k_{B}$ is the Boltzmann constant. The total specific heat of the crystal is the sum of all phonon modes over the Brillouin zone (BZ),

$$
C_{v}(T)=\sum_{j, \mathbf{q}} c_{v, j}(\mathbf{q}, T) .
$$

$c_{v, j}(\mathbf{q}, T)$ is the mode contribution to the specific heat defined as,

$$
c_{v, j}(\mathbf{q}, T)=k_{B} \sum_{j, \mathbf{q}}\left(\frac{\hbar \omega_{j}(\mathbf{q}, V)}{2 k_{B} T}\right)^{2} \frac{1}{\sinh ^{2}\left[\hbar \omega_{j}(\mathbf{q}, V) / 2 k_{B} T\right]} .
$$

The mode Grüneisen parameter $\gamma_{j}(\mathbf{q})$ describing the phonon frequency shift with respect to the volume can be calculated by

$$
\gamma_{j}(\mathbf{q})=-\frac{d\left[\ln \omega_{j}(\mathbf{q}, V)\right]}{d[\ln V]} .
$$

The acoustic Grüneisen parameter $\gamma(T)$ defined as the weighted average of the mode Grüneisen parameters for all acoustic phonon branches is calculated to be

$$
\gamma(T)=\frac{\alpha_{v}(T) B(T) V_{m}(T)}{C_{v}(T)},
$$

where $\alpha_{v}(T)$ is the thermal expansion coefficient and equals to $\frac{1}{V}\left(\frac{\partial V}{\partial T}\right)_{P}, V_{m}(T)$ is the volume per mole material, and $B(T)$ and $C_{v}(T)$ are the bulk modulus and specific heat respectively.

The formation energy of a point defect $\mathrm{X}$ with charges $q$ can be calculated by introducing the chemical potential concept as,

$$
E_{\text {for }}\left(\mathrm{X}^{q}\right)=E_{t o t} \pm n_{x} \mu-E_{\mathrm{ThO}_{2}}+q\left(E_{v}+E_{f}+\Delta V\right),
$$

where $E_{t o t}$ is the total energies of the supercell with defect $\mathrm{X}, n_{x}$ represents the number of $\mathrm{X}$ defects, $\mu$ is the chemical potential of $\mathrm{X}$ with a positive sign for vacancy and a negative sign for interstitial defect, $E_{\mathrm{ThO}_{2}}$ is the energy of the $\mathrm{ThO}_{2}$ supercell without defects, $E_{v}$ and $\mathrm{E}_{f}$ are the valence-band maximum (VBM) and the Fermi level of $\mathrm{ThO}_{2}$ respectively. With these denotations, $E_{\text {for }}\left(\mathrm{V}_{\mathrm{O}}^{0}\right), E_{\text {for }}\left(\mathrm{O}_{i}^{0}\right), E_{\text {for }}\left(\mathrm{V}_{\mathrm{Th}}^{0}\right)$, and $E_{\text {for }}\left(\mathrm{Th}_{i}^{0}\right)$ represent for the formation energies of a neutral oxygen vacancy, a neutral interstitial oxygen, a neutral thorium vacancy, and a neutral interstitial thorium respectively. The shift of the VBM in a defect supercell $\Delta V$ takes the change of the valence-band maximum caused by the defect into account. Its value can be obtained by a macroscopic average technique [42, 43] through calculating the difference between the average electrostatic potential in a bulklike environment of the defect supercell and the average electrostatic potential in the defect-free supercell. The formation energy of a Frenkel-pair can be calculated by summing up the formation energies of a vacancy and a corresponding interstitial add-in, i.e.,

$$
E_{\text {for }}\left(\mathrm{FP}_{\mathrm{X}}\right)=E_{\text {for }}\left(\mathrm{V}_{\mathrm{X}}\right)+E_{\text {for }}\left(\mathrm{X}_{\mathrm{i}}\right) .
$$

For the Schottky defect, the formation energy can be calculated by

$$
E_{f o r}(\mathrm{~S})=E_{f o r}\left(\mathrm{~V}_{\mathrm{Th}}\right)+2 E_{f o r}\left(\mathrm{~V}_{\mathrm{O}}\right)-\frac{3(N-1)}{N} E_{\mathrm{ThO}_{2}}
$$

where $N$ is the number of atoms in the considered supercell. In this expression, the defect consists of a thorium vacancy and two oxygen vacancies, which are again supposed to be non-interacting. 


\section{RESULTS AND DISCUSSIONS}

\section{A. Structure and Elastic constants of $\mathbf{T h O}_{2}$}

Our previous studies have shown that DFT calculations with GGA exchange-correlation functionals are good enough for obtaining the ground-state properties of $\mathrm{ThO}_{2}$ [47], and adding additional $U$ or $J$ modifications for localization effects might lead to incorrect results. We believe that it is because the $5 f$ electronic states of Th become delocalized after electronic hybridizations in $\mathrm{ThO}_{2}$. Here based on our previous studies, we further investigate the thermodynamic properties of $\mathrm{ThO}_{2}$.

From the mechanical point of view, the theoretical equilibrium volume $V_{0}$, bulk modulus $B_{0}$, and the pressure derivative of bulk modulus $B^{\prime}$ can be obtained by fitting the third-order Brich-Murnaghan equation of state [44]. In this way, our calculated lattice parameter $\mathrm{a}_{0}$ for $\mathrm{ThO}_{2}$ is $5.619 \AA$, which is in accordance with the experimental data of $5.60 \AA$ [29, 45]. The bulk modulus $B_{0}$ and its pressure derivative $B^{\prime}$ are calculated to be 190 GPa and 4.3, also in agreement with corresponding experimental values of 195-198 GPa and 4.6-5.4 [29, 45], respectively. In order to evaluate the Poisson's ratio $\nu$, we calculated the three independent elastic constants $C_{11}$, $C_{12}$ and $C_{44}$ of $\mathrm{ThO}_{2}$. The calculation methods are the same as in our previous studies [35, 46, 47]. The obtained elastic constants for $\mathrm{ThO}_{2}$ are $C_{11}=351.2 \mathrm{GPa}$, $C_{12}=106.9 \mathrm{GPa}$, and $C_{44}=74.1 \mathrm{GPa}$, which are in accordance with experimentally measured values of $C_{11}=367$ $\mathrm{GPa}, C_{12}=106 \mathrm{GPa}$, and $C_{44}=79 \mathrm{GPa}$ 48]. Furthermore, the Poisson's ratio $\nu$ is calculated to be 0.293 , in excellent accordance with the experimental data of 0.285 [48]. All the good agreement between our calculated values and corresponding experimental measurements indicates that our calculation methods are effective and reliable. In comparison with other actinide dioxides, we can see that $\mathrm{ThO}_{2}$ has a slightly smaller bulk modulus than $\mathrm{UO}_{2}$ and $\mathrm{PuO}_{2}$ [47.

\section{B. Phonon dispersions, Thermal expansion, and Heat capacity of $\mathrm{ThO}_{2}$}

The thermodynamic properties of a material are connected to its phonon dispersion curves. In order to get accurate splittings between longitudinal (LO) and transverse optical (TO) phonon branches, the Born effective charge is firstly calculated. Because of the high symmetry of $\mathrm{ThO}_{2}$, the Born transverse effective charge tensor $\mathbf{Z}^{*}$ is the same along the [100], [010], and [001] directions, and the effective charge can be averaged by $Z^{*}=\frac{1}{3} \operatorname{Tr} \mathbf{Z}^{*}$. For $\mathrm{ThO}_{2}$, we obtain that $Z_{\mathrm{Th}}^{*}=+5.41$, and $Z_{\mathrm{O}}^{*}=-2.71$. The static dielectric constant $\epsilon=\frac{1}{3} \operatorname{Tr} \epsilon$ is 4.83. The dielectric constant might be smaller than experimental measurements because of the underestimation of the electronic energy band gap due to the drawback of the exchange-correlation approximation (GGA).

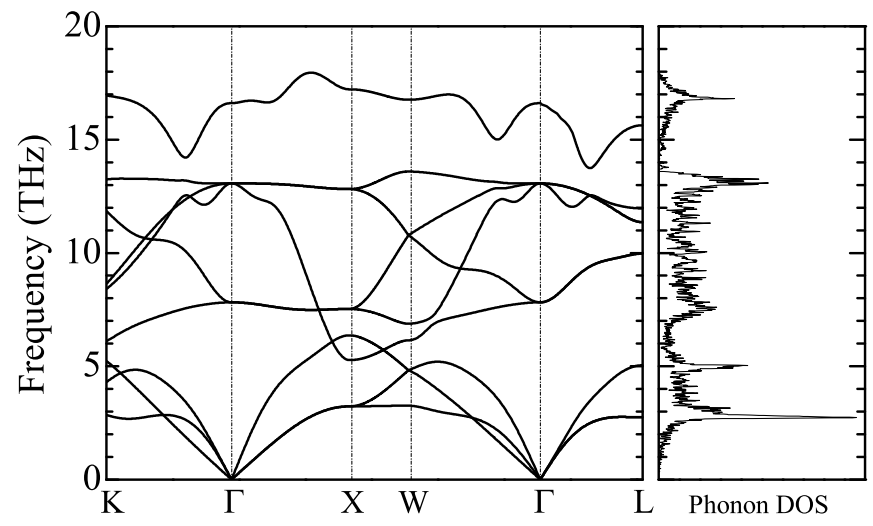

FIG. 1: (Color online) Phonon dispersion curves and phonon density of states (DOS) for $\mathrm{ThO}_{2}$.

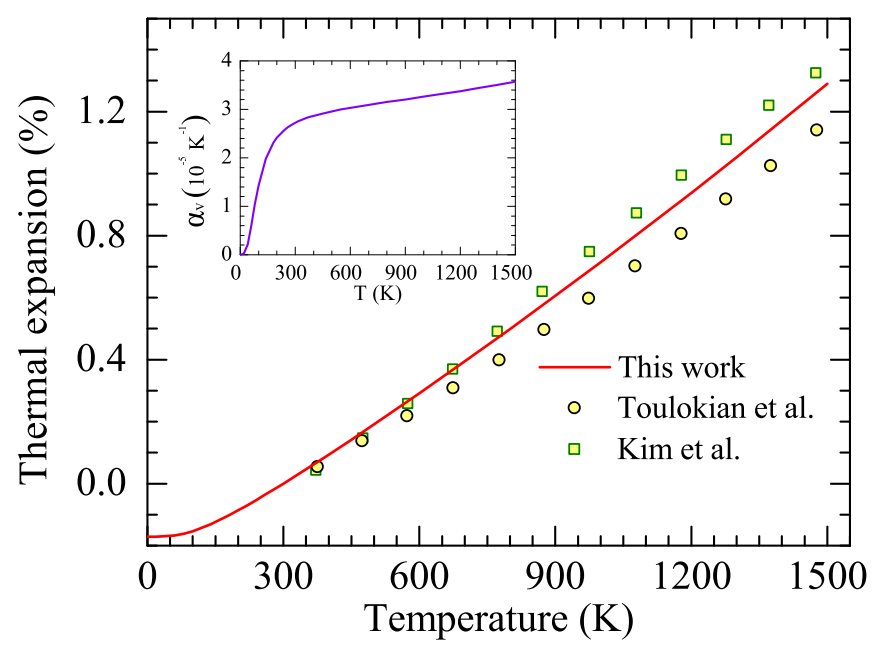

FIG. 2: (Color online) Temperature dependence of the linear thermal expansion for $\mathrm{ThO}_{2}$. The inset is the volume thermal expansion coefficient as a function of temperature.

The calculated phonon dispersion curves along the highsymmetry k-point lines using the above Born effective charges are shown in Fig. 1. As clearly shown, there is an obvious splitting between longitudinal optical (LO) and TO branches due to the Born effective charge. Moreover, we find that there is no evident gap between acoustic and optical branches of phonon for $\mathrm{ThO}_{2}$, with an observable overlap between the longitudinal acoustic (LA) and transverse optical (TO) branches around the $\mathrm{X}$ point. Detailed vibrational modes analysis tells us that the vibrations of $\mathrm{Th}$ and $\mathrm{O}$ atoms dominate the low- $(0-6 \mathrm{THz})$ and high-frequency $(6-18 \mathrm{THz})$ modes, respectively.

From the obtained phonon dispersion curves and Eqs. (1) and (2), we then calculate the energy curves for $\mathrm{ThO}_{2}$, and find the lowest-energy lattice constants at different temperatures. The lattice expansion curve is thus obtained and shown in Fig. 2. The experimental data by Touloukian et al. [49] and Kim et al. [30] are also shown 


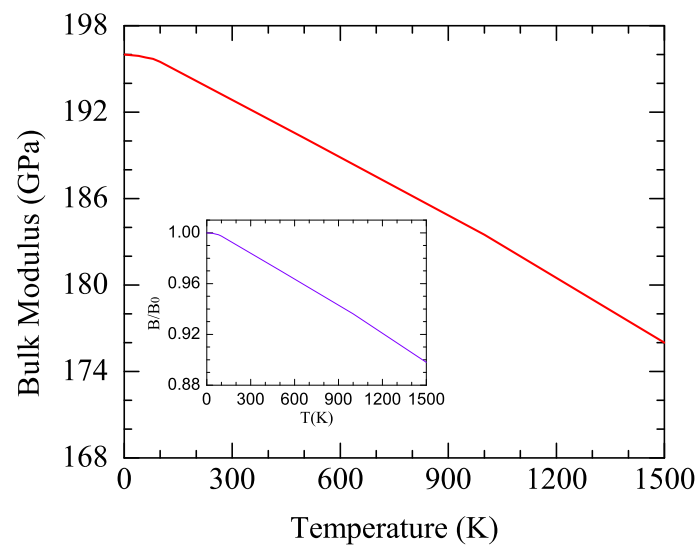

FIG. 3: (Color online) Temperature dependence of bulk modulus $B$ for $\mathrm{ThO}_{2}$. The inset is the ratio of $B / B_{0}$.

in Fig. 2 for comparisons with our calculational results. One can see that in the temperature range from 300 to $600 \mathrm{~K}$, our result is in excellent agreement with the experimental data. In a higher temperature range from 600 to $1500 \mathrm{~K}$, our obtained theoretical values are in the middle of the two different experimental measurements. And at $1500 \mathrm{~K}$, the relative difference between our result and the two experimental values are $0.15 \%$ and $0.05 \%$, respectively. The small relative differences indicate that the QHA method can give reasonable lattice parameters for $\mathrm{ThO}_{2}$ up to $1500 \mathrm{~K}$. The thermal expansion coefficient $\alpha_{v}(T)$ is calculated and shown in the inset of Fig. 2. The experimental values for the thermal expansion coefficient in the temperature range from 298 to $1500 \mathrm{~K}$ can be calculated from the corresponding thermal expansion curves, which are $3.318 \times 10^{-5} \mathrm{~K}^{-1}$ for Touloukian's data and $3.630 \times 10^{-5} \mathrm{~K}^{-1}$ for Kim's data, respectively. Our theoretical result of $3.509 \times 10^{-5} K^{-1}$ is consistent with the experimental values.

The bulk modulus $B$ is also analyzed as a function of temperature according to the formula $B=V_{0}\left(\frac{\partial^{2} F}{\partial V^{2}}\right)_{V_{0}}$, and the result is displayed in Fig. 3. We can see that the value of bulk modulus decreases with increasing temperature, and at $1500 \mathrm{~K}$, the ratio of bulk modulus $\left(B / B_{0}\right)$ is 0.898 , as depicted in the inset of Fig. 3.

Under QHA, the considered vibration modes are harmonic but volume-dependent. The calculated heat capacity at constant volume using Eqs. (3) and (4) is shown in Fig. 4, together with the heat capacity at constant pressure $C_{p}$, which is calculated according to the relationship,

$$
C_{p}-C_{v}=\alpha_{v}^{2}(T) B(T) V(T) T .
$$

The available experimental data from Bakker et al. 23. and Kim et al. [30] are also shown in Fig. 4 for comparisons. It is evident that our theoretical result is in excellent agreement with the measured values for the whole experimentally considered temperature range. As temperature increases, the value of $C_{p}$ increases continuously,

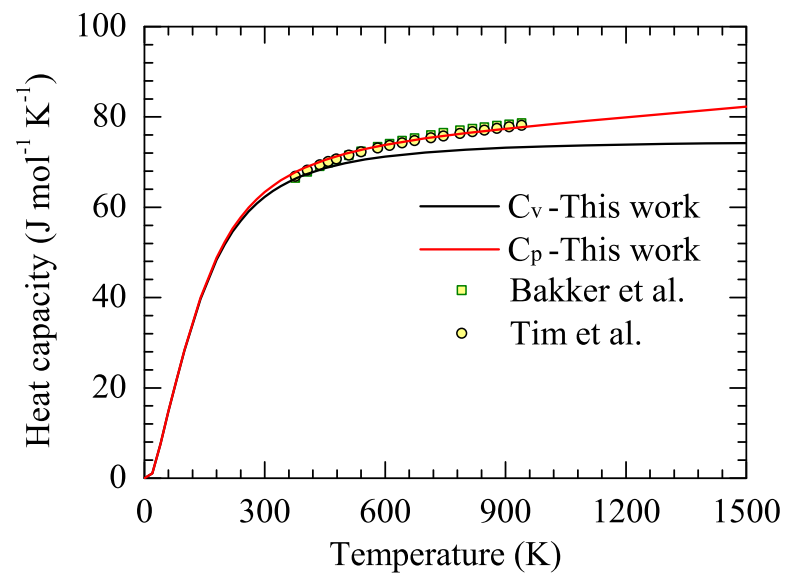

FIG. 4: (Color online) Heat capacities at constant volume $\left(C_{v}\right)$ and constant pressure $\left(C_{p}\right)$ of $\mathrm{ThO}_{2}$.

while the value of $C_{v}$ approaches to a constant of $3 R(R$ is the Rydberg constant). At $1500 \mathrm{~K}$, the value of $C_{p}$ becomes $81 \mathrm{~J} \cdot \mathrm{mol}^{-1} \mathrm{~K}^{-1}$. In general, QHA method is valid when the temperature is much lower than the material's melting point (around $3600 \mathrm{~K}$ for $\mathrm{ThO}_{2}[23]$ ) when the anharmonic effect is small.

\section{Grüneisen parameters and thermal conductivity of $\mathrm{ThO}_{2}$}

The lattice thermal conductivity $\kappa_{L}$ for a material can be calculated differently, depending on the specific mechanisms for phonon scattering. At relative high temperatures, the dominant mechanism for phonon scattering is the Umklapp process, in which the acoustic phonon branches interact with each other and transport heat. With this mechanism, the lattice thermal conductivity of a crystal-like material can be expressed as 50 52],

$$
\kappa_{L}=A \frac{\bar{M} \Theta^{3}(T) \delta(T) n^{2 / 3}}{\gamma^{2}(T) \times T}
$$

where $A$ is a physical constant with the value of $3.1 \times 10^{-6}, \bar{M}$ is the average mass per atom in the crystal, $\Theta(T)$ is the Debye temperature of $\mathrm{ThO}_{2}, n$ is the number of atoms in the primitive unit cell, $\gamma(T)$ is the acoustic Grüneisen parameter, and $\delta(T)$ is the cube root of the average volume per atom, i.e., the averaged radius per atom. The $\kappa_{L}$ and $\delta$ in Eq. (11) are in units of $\mathrm{W}$ $\mathrm{m}^{-1} \mathrm{~K}^{-1}$, and $\AA$ respectively. With reasonable expressions of the Debye temperature and acoustic Grüneisen parameter to describe the harmonic phonon branches and the anharmonic interactions between different phonon branches, Eq. (11) can provide accurate predictions for a material's thermal conductivity [51].

Firstly the Debye temperature $\Theta$ can be determined from the elastic constants within the Debye theory, in which the vibrations of the solid are considered as elastic 


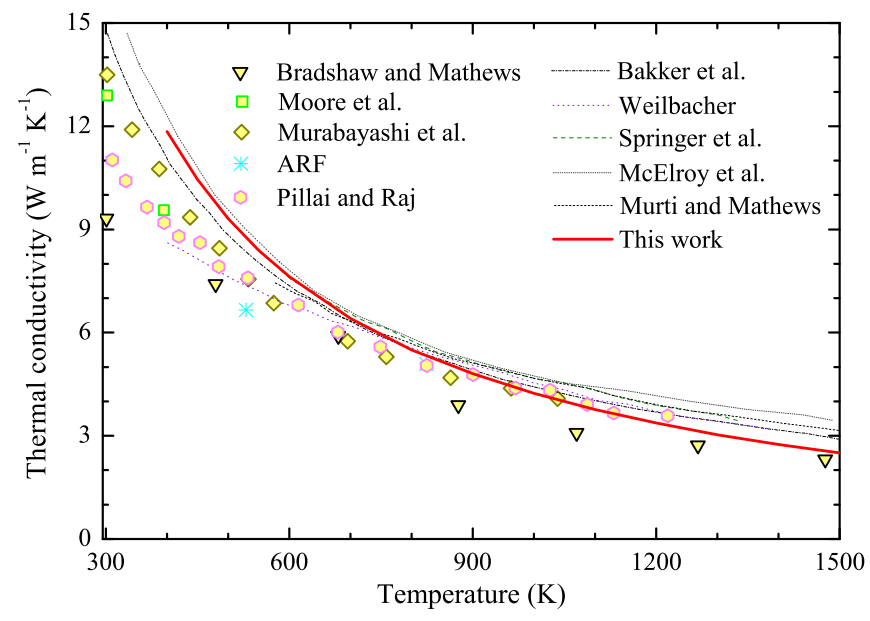

FIG. 5: (Color online) The thermal conductivity of $\mathrm{ThO}_{2}$. Experimental results from Springer et al. [14], Weibacher [15], McElroy et al. [16], ARF [17], Murabayashi et al. [18], Pillai and Raj 19], Bradshaw 20], Moore et al. 21], Murti and Mathews [22], and Bakker et al. [23] are displayed for comparison.

waves, and the Debye temperature of the solid is related to an averaged sound velocity [53]. Within isotropic approximation, the Debye temperature $\Theta$ can be expressed as [53],

$$
\Theta(T)=\frac{\hbar}{k_{B}}\left[6 \pi^{2} V^{1 / 2}(T) n\right]^{1 / 3} f(\nu) \sqrt{\frac{B(T)}{M}},
$$

where $\mathrm{M}$ is the molecular mass per formula unit, $B(T)$ is the bulk modulus, $\nu$ is the material's Poisson's ratio, and $f(\nu)$ is given by $[54,55]$

$$
f(\nu)=3^{1 / 3}\left[2\left(\frac{2}{3} \frac{1+\nu}{1-2 \nu}\right)^{3 / 2}+\left(\frac{1}{3} \frac{1+\nu}{1-\nu}\right)^{3 / 2}\right]^{-1 / 3} .
$$

Our calculations find that the Debye temperature monotonically decreases, while the acoustic Grüneisen parameter monotonically increases with increasing temperature.

Based on Eq. (11) and the obtained Debye temperature, we calculate the thermal conductivity for $\mathrm{ThO}_{2}$. Figure 5 shows our thermal conductivity result, in comparison with the previous experimental measurements in the temperature range from 300 to $1500 \mathrm{~K}$. We can see that in the considered temperature range, our calculated values are in agreement with the experimental results, which proves the validity of our methods and model. Especially, in the high temperature range from 600 to 1200 $\mathrm{K}$, our results accords very well with the experimental measurements by Murabayashi, et al. [18] and by Pillai and Raj [19]. At the low temperature range around the Debye temperature of $402 \mathrm{~K}$, our results are slightly different from some experimental results. This difference comes from our presumption that the dominant mechanism for phonon scattering is the Umklapp process. The

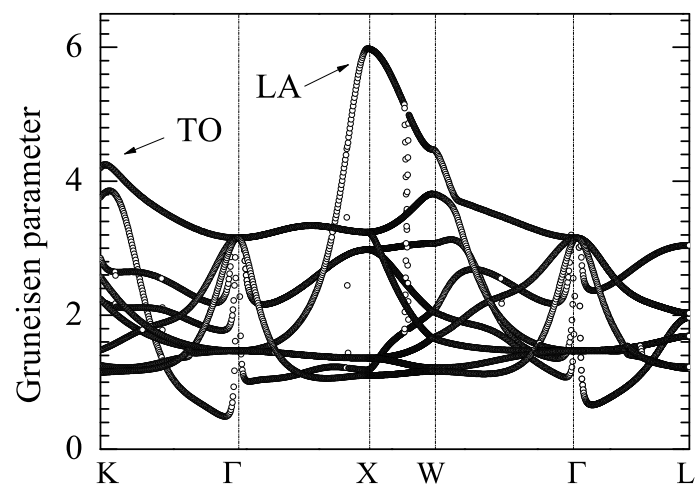

FIG. 6: (Color online) The mode Grüneisen parameters along high-symmetry directions in the reciprocal lattice space of $\mathrm{ThO}_{2}$.

accordance between our calculations and corresponding experiments in the temperature range from 600 to 1200 $\mathrm{K}$ proves that within this temperature area, the contributions from other phonon-scattering mechanisms are so small that can be neglected.

The mode Grüneisen parameter describing the phonon frequency shift with respect to the volume can be used to discuss the anharmonic effects. By expanding or compressing the equilibrium volume by $1 \%$, we calculate the mode Grüneisen parameter $\gamma_{j}(\mathbf{q})$ for all nine phonon branches according to Eq. (5). The corresponding results are shown in Fig. 6. It can be seen that all the mode Grüneisen parameter values are positive, indicating that all phonon frequencies increase with decreasing volume. Besides, the acoustic phonon mode Grüneisen parameters are relatively larger reflecting that changes in volume have more influences on the collective vibration modes of $\mathrm{ThO}_{2}$. We can also see from Fig. 6 that the LA and TO phonon branches have larger mode Grüneisen parameters, indicating that the anharmonic interactions between the LA and TO branches should be more intensive with respect to the volume change.

\section{Defect formation in $\mathrm{ThO}_{2}$}

In this subsection and the next, we will investigate the structural stability of $\mathrm{ThO}_{2}$ by systematically calculating the formation energy of different kinds of defects and investigating the diffusion behaviors of helium. A $2 \times 2 \times 2$ supercell with $96 \mathrm{Th}$ and $\mathrm{O}$ atoms is employed in these two subsections to model defect formation and helium diffusion in $\mathrm{ThO}_{2}$. Different charge states are considered for all the defects. To calculate the formation energy, the positions of all ions are fully relaxed before we calculate the electronic free energies of the system with different defects.

The formation energy for oxygen (thorium) vacancies, interstitial oxygen (thorium) ions, oxygen (thorium) Frenkel pairs, and the Schottky defect of a $\mathrm{ThO}_{2}$ unit in 
TABLE I: Formation energies of different defects at different charge states in $\mathrm{ThO}_{2}$. The defects include oxygen vacancy ( $\mathrm{V}_{\mathrm{O}}$ ), interstitial oxygen ion $\left(\mathrm{O}_{i}\right)$, thorium vacancy $\left(\mathrm{V}_{\mathrm{Th}}\right)$, interstitial thorium ion $\left(\mathrm{Th}_{i}\right)$, oxygen $\left(\mathrm{FP}_{\mathrm{O}}\right)$ and thorium Frenkel-pairs $\left(\mathrm{FP}_{\mathrm{Th}}\right)$, and Schottky defect $(\mathrm{S})$ of a $\mathrm{ThO}_{2}$ unit. The formation energies are in units of eV.

\begin{tabular}{|c|c|c|c|}
\hline Defect & Charge on defect & Kröger-Vink notation & $E_{\text {for }}$ \\
\hline $\mathrm{V}_{\mathrm{O}}$ & 0 & $\mathrm{~V}_{\mathrm{O}}^{\mathrm{X}}$ & 7.415 \\
\hline $\mathrm{V}_{\mathrm{O}}$ & +1 & $\mathrm{~V}_{\mathrm{O}}^{\bullet}$ & 3.922 \\
\hline $\mathrm{V}_{\mathrm{O}}$ & +2 & $\mathrm{~V}_{\mathrm{O}}^{\bullet \bullet}$ & 1.338 \\
\hline $\mathrm{O}_{i}$ & 0 & $\mathrm{O}_{i}^{\mathrm{X}}$ & 1.901 \\
\hline $\mathrm{O}_{i}$ & -1 & $\mathrm{O}_{i}^{\prime}$ & 4.026 \\
\hline $\mathrm{O}_{i}$ & -2 & $\mathrm{O}_{i}^{\prime \prime}$ & 5.487 \\
\hline $\mathrm{V}_{\mathrm{Th}}$ & 0 & $\mathrm{~V}_{\mathrm{Th}}^{\mathrm{X}}$ & 18.349 \\
\hline $\mathrm{V}_{\mathrm{Th}}$ & -1 & $\mathrm{~V}_{\mathrm{Th}}^{\prime}$ & 18.600 \\
\hline $\mathrm{V}_{\mathrm{Th}}$ & -2 & $\mathrm{~V}_{\mathrm{Th}}^{\prime \prime}$ & 18.314 \\
\hline $\mathrm{V}_{\mathrm{Th}}$ & -3 & $\mathrm{~V}_{\mathrm{Th}}^{\prime \prime \prime \prime}$ & 18.327 \\
\hline $\mathrm{V}_{\mathrm{Th}}$ & -4 & $\mathrm{~V}_{\mathrm{Th}}^{\prime \prime \prime \prime}$ & 18.462 \\
\hline $\mathrm{Th}_{i}$ & 0 & $\operatorname{Th}_{i}^{\mathrm{X}}$ & 6.094 \\
\hline $\mathrm{Th}_{i}$ & +1 & $\mathrm{Th}_{i}^{\bullet}$ & 5.897 \\
\hline $\mathrm{Th}_{i}$ & +2 & $\mathrm{Th}_{i}^{\bullet \bullet}$ & 2.869 \\
\hline $\mathrm{Th}_{i}$ & +3 & $\mathrm{Th}_{i}^{\bullet \bullet \bullet}$ & 0.488 \\
\hline $\mathrm{Th}_{i}$ & +4 & $\mathrm{Th}_{i}^{\bullet \bullet \bullet \bullet}$ & -1.741 \\
\hline $\mathrm{FPO}_{\mathrm{O}}$ & 0 & $\mathrm{~V}_{\mathrm{O}}^{\mathrm{X}}+\mathrm{O}_{i}^{\mathrm{X}}$ & 9.316 \\
\hline $\mathrm{FP}_{\mathrm{O}}$ & 0 & $\mathrm{~V}_{\mathrm{O}}^{\bullet}+\mathrm{O}_{i}^{\prime}$ & 7.948 \\
\hline $\mathrm{FP}_{\mathrm{O}}$ & 0 & $\mathrm{~V}_{\mathrm{O}}^{\bullet \bullet}+\mathrm{O}_{i}^{\prime \prime}$ & 6.825 \\
\hline $\mathrm{FP}_{\mathrm{Th}}$ & 0 & $\mathrm{~V}_{\mathrm{Th}}^{\mathrm{X}}+\mathrm{Th}_{i}^{\mathrm{X}}$ & 24.443 \\
\hline $\mathrm{FP}_{\mathrm{Th}}$ & 0 & $\mathrm{~V}_{\mathrm{Th}}^{\prime}+\mathrm{Th}_{i}^{\bullet}$ & 24.497 \\
\hline $\mathrm{FP}_{\mathrm{Th}}$ & 0 & $\mathrm{~V}_{\mathrm{Th}}^{\prime \prime}+\mathrm{Th}_{i}^{\bullet \bullet}$ & 21.183 \\
\hline $\mathrm{FP}_{\mathrm{Th}}$ & 0 & $\mathrm{~V}_{\mathrm{Th}}^{\prime \prime \prime}+\mathrm{Th}_{i}^{\bullet \bullet \bullet}$ & 18.815 \\
\hline $\mathrm{FP}_{\mathrm{Th}}$ & 0 & $\mathrm{~V}_{\mathrm{Th}}^{\prime \prime \prime \prime}+\mathrm{Th}_{i}^{\bullet \bullet \bullet \bullet ~}$ & 16.721 \\
\hline $\mathrm{S}$ & 0 & $\mathrm{~V}_{\mathrm{Th}}^{\mathrm{X}}+2 \mathrm{~V}_{\mathrm{O}}^{\mathrm{X}}$ & 19.472 \\
\hline $\mathrm{S}$ & 0 & $\mathrm{~V}_{\mathrm{Th}}^{\prime \prime}+2 \mathrm{~V}_{\mathrm{O}}^{\bullet}$ & 12.651 \\
\hline $\mathrm{S}$ & 0 & $\mathrm{~V}_{\mathrm{Th}}^{\prime \prime \prime \prime}+2 \mathrm{~V}_{\mathrm{O}}^{\bullet \bullet}$ & 8.231 \\
\hline
\end{tabular}
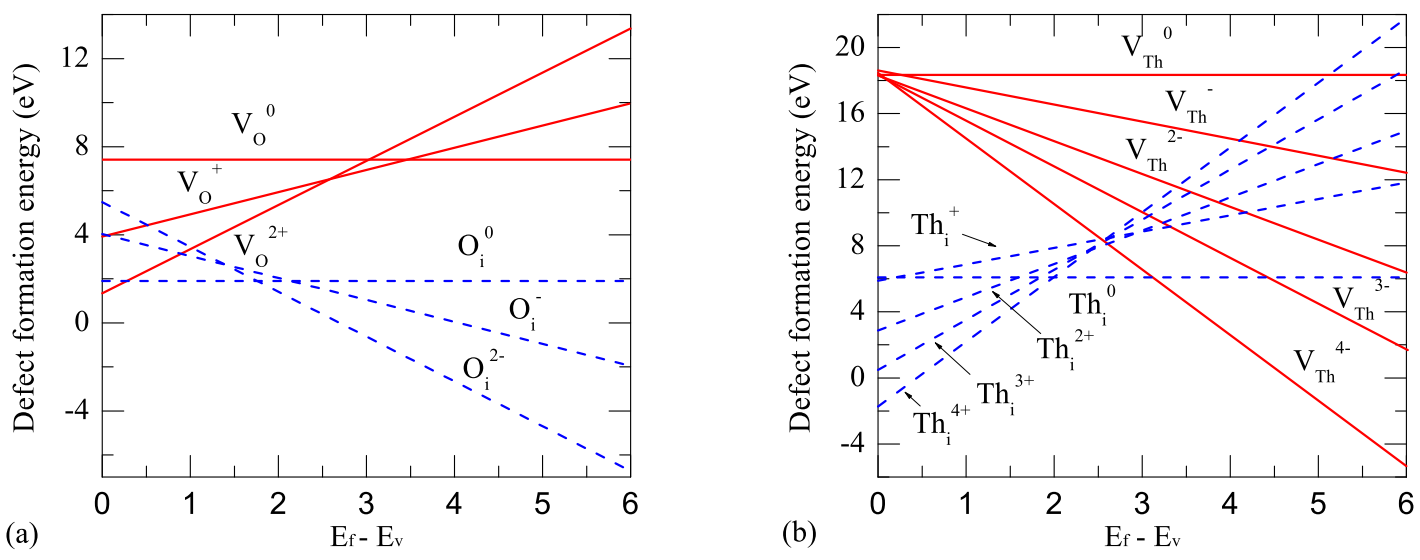

FIG. 7: (Color online) Formation energies of oxygen related defects, i.e., oxygen vacancies or interstitial oxygen ions (a) and thorium related defects, i.e., thorium vacancies or interstitial thorium ions (b) as functions of the Fermi energy.

the $2 \times 2 \times 2$ supercell are obtained and listed in Table 1 One can see that the charged $\mathrm{Th}_{i}^{4+}$ add-in defect is the only one having a negative formation energy. It means that once $\mathrm{Th}_{i}^{4+}$ ions are available, interstitial $\mathrm{Th}_{i}^{4+}$ defect can form in $\mathrm{ThO}_{2}$ with an energy release of $1.74 \mathrm{eV}$.
Comparatively for oxygen defects, the $\mathrm{O}^{2-}$ vacancy is the most possible one because of its smallest formation energy among oxygen defects. These two results reflects the ionic character of the Th-O bonds that each thorium atom almost loses 4 electrons to two oxygen atoms 

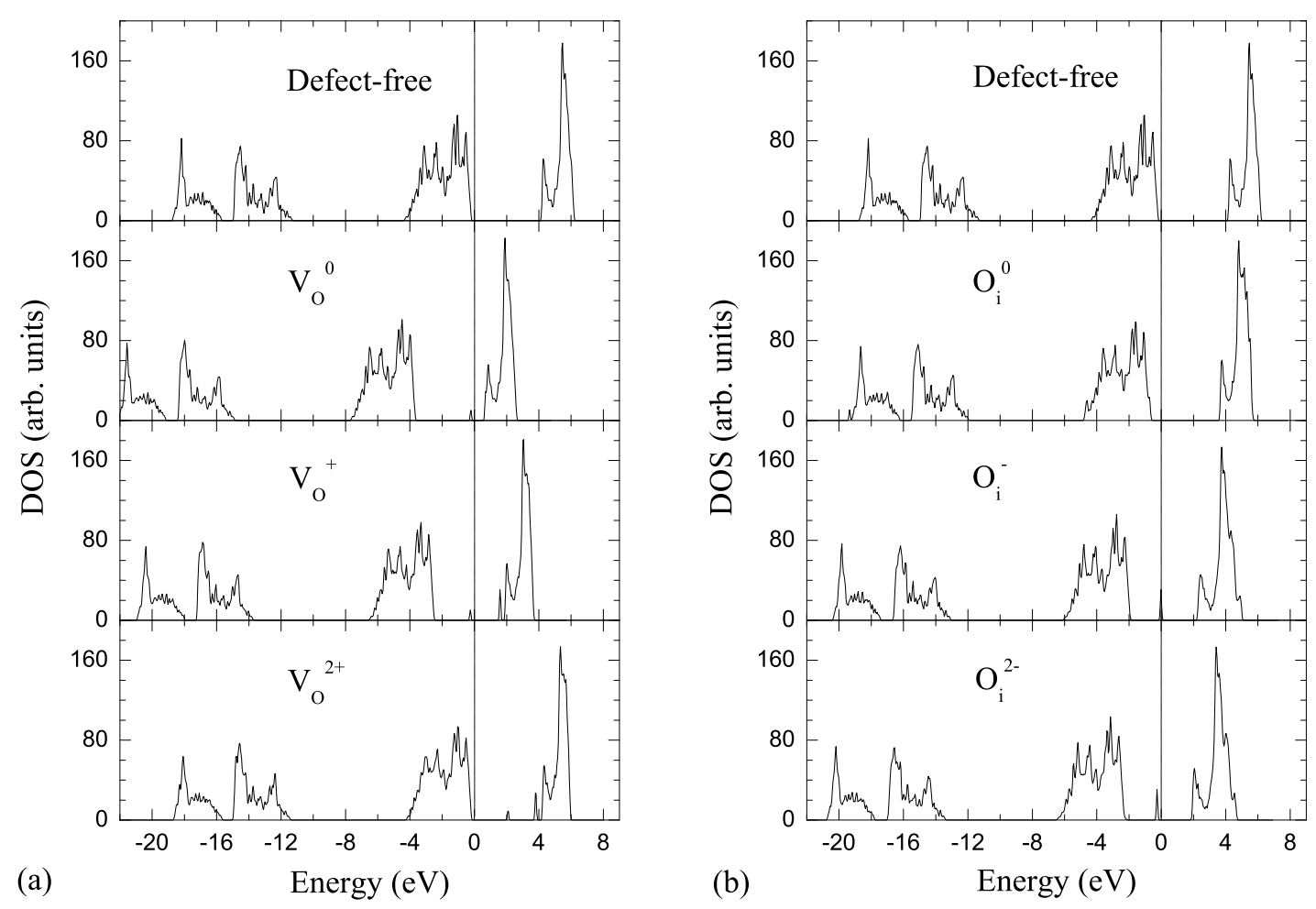

FIG. 8: (Color online) Electronic density of states (DOS) for $\mathrm{ThO}_{2}$ with oxygen vacancies (a), and with interstitial oxygen defects (b) in different charge states. The Fermi energies are set to zero.

in $\mathrm{ThO}_{2}$. From Table I, we can also see that all thorium vacancies and thorium Frenkel-pairs have huge formation energies and thus can hardly form in intrinsic $\mathrm{ThO}_{2}$. Comparatively, the formation of oxygen Frenkelpair, containing an $\mathrm{O}^{2+}$ vacancy and a interstitial $\mathrm{O}^{2-}$ ion has a relatively smaller formation energy.

Depending on the environment's influence on the Fermi energy of the $\mathrm{ThO}_{2}$ material, the formation possibility of charged defects can change due to the changes on hardness of electrons transfer. Using Eqs. (7)-(9) under different Fermi energies, we can calculate the formation energy as a function of the Fermi energy for different kinds of defects. The corresponding results for oxygen (thorium) vacancies and interstitial oxygen (thorium) ions in different charge states are respectively shown in Figs. 7(a) and 7 (b). The experimentally measured band-gap of $6.0 \mathrm{eV}$, instead of the $4.1 \mathrm{eV}$ value obtained by first-principles calculations is chosen as the reference band-gap in discussions on defect formation.

As can be seen from Fig. 7(a), when comparing the energies associated with forming $\mathrm{V}_{\mathrm{O}}^{0}, \mathrm{~V}_{\mathrm{O}}^{+}$, and $\mathrm{V}_{\mathrm{O}}^{2+}$ oxygen vacancies, a transition can be observed with the Fermi level increasing from the valence band to the conduction band. The +2 charged oxygen vacancy is favored near the valence band, indicating that oxygen vacancies have a tendency to donate electrons or behave as a $n$-type defect. When the Fermi level increases to around $2.6 \mathrm{eV}$, the $\mathrm{V}_{\mathrm{O}}^{+}$becomes energetically favorable. With further in- creasing the Fermi level, the neutral charge state is most probable for oxygen vacancies, and the tendency of oxygen vacancy to donate electrons diminishes. We can also see from Fig. $7\left(\right.$ a) that the interstitial $\mathrm{O}_{i}^{2-}$ defect can become very possible to form when the Fermi energy is shifted to be near the conduction band of $\mathrm{ThO}_{2}$.

From Fig. 7(b) we see that when the Fermi energy is near the valence band, all charged states of thorium vacancy are hard to form because of the huge formation energies, and the interstitial $\mathrm{Th}_{i}^{4+}$ ions can easily form in $\mathrm{ThO}_{2}$ with a negative formation energy. With upshifting the Fermi energy, the interstitial thorium defect in neutral state and the $\mathrm{V}_{\mathrm{Th}}^{4-}$ vacancy can both become the most possible thorium kinds of defects. When considering only the vacancy defects, $\mathrm{V}_{\mathrm{O}}^{2+}$ is the most stable defect near the valence band, while near the conduction band, $\mathrm{V}_{\mathrm{Th}}^{4-}$ is the most favorable one. Comparatively for interstitial states, the $\mathrm{Th}_{i}^{4+}$ and $\mathrm{O}_{i}^{2-}$ defects are the most probable ones when the Fermi energy is near the valence and conduction band respectively.

In addition, the electronic density of states (DOS) for both defect-free and defective $\mathrm{ThO}_{2}$ are calculated and shown in Figs. 8 and 9, to further analyze the influences of the considered defects on the electronic structures of $\mathrm{ThO}_{2}$. As clearly shown in both Figs. 8 and 9, the introduction of vacancies or interstitial ions do not change the DOS distribution of $\mathrm{ThO}_{2}$ very much. The biggest character in both Figs. 8 and 9 is that a new defect en- 

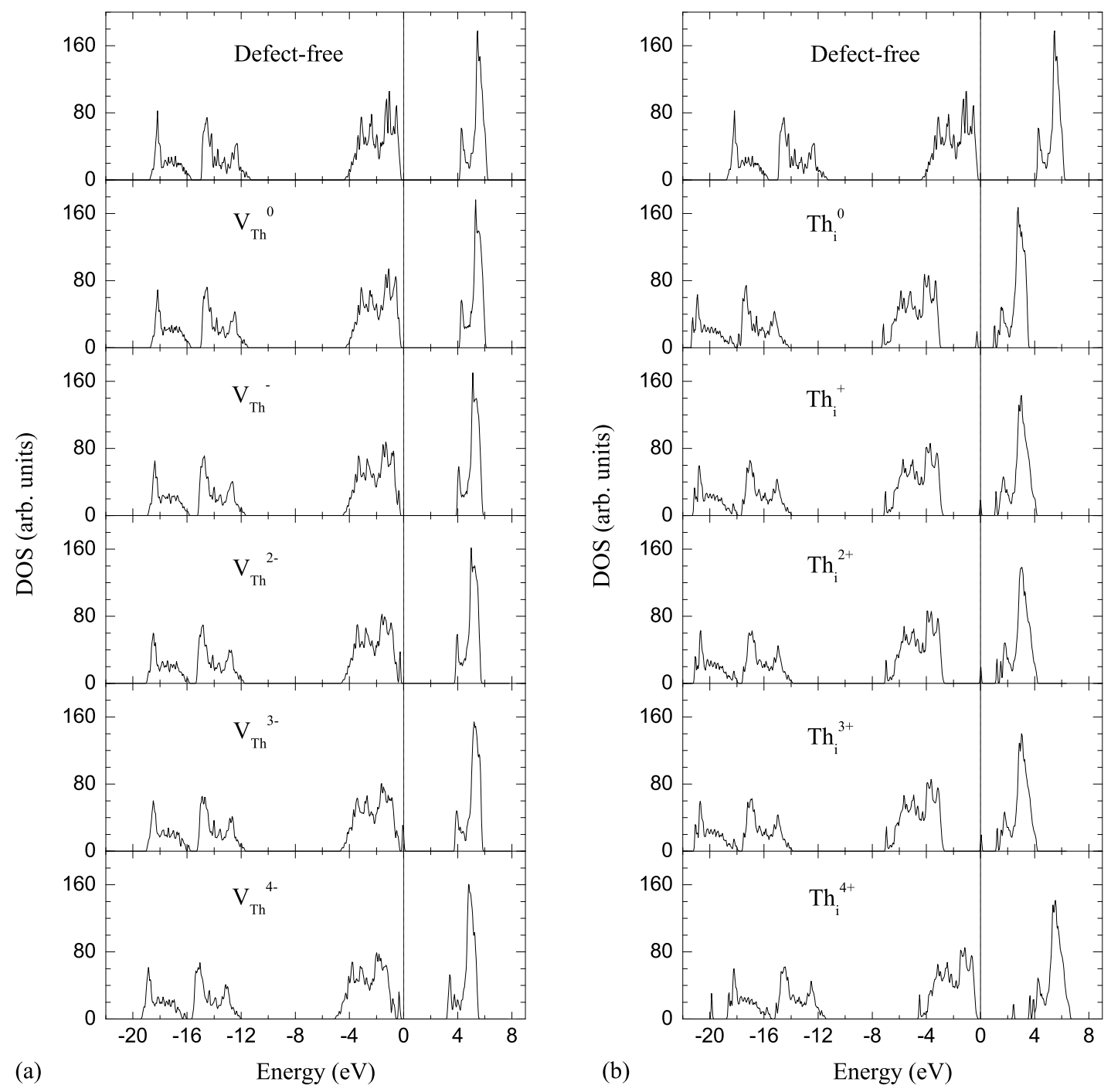

FIG. 9: (Color online) Electronic density of states (DOS) for $\mathrm{ThO}_{2}$ with thorium vacancies (a), and with interstitial thorium defects (b) in different charge states. The Fermi energies are set to zero.

ergy level emerges in the band gap for defective $\mathrm{ThO}_{2}$. For the electronic structure of $\mathrm{ThO}_{2}$ with oxygen vacancies, we see from Fig. 8(a) that the Fermi energy shifts from the valence band maximum (VBM) in defect-free $\mathrm{ThO}_{2}$, to above the defect energy level for the $\mathrm{V}_{\mathrm{O}}^{0}$ defect, and from above the defect energy level for the $\mathrm{V}_{\mathrm{O}}^{+}$defect back to the VBM for the $\mathrm{V}_{\mathrm{O}}^{2+}$ defect. For the system with interstitial oxygen ions, we can see from Fig. 8(b) that the Fermi energy shifts respectively from the VBM for the $\mathrm{O}_{i}^{0}$ defect to the defect energy level for the $\mathrm{O}_{i}^{-}$ defect, and from the defect energy level for the $\mathrm{O}_{i}^{-}$defect to above the defect energy level for the $\mathrm{O}_{i}^{2-}$ defect. Similarly for the $\mathrm{ThO}_{2}$ supercell with interstitial thorium ions, the Fermi energy shifts from the VBM to the defect energy level for the $\mathrm{Th}_{i}^{+}, \mathrm{Th}_{i}^{2+}$, and $\mathrm{Th}_{i}^{3+}$ defects, and then shifts to above the defect energy level for the $\mathrm{Th}_{i}^{4+}$ defect, as shown in Fig. 9(b). From Fig. 9(a), one can see that the defect energy levels for thorium vacancies in $\mathrm{ThO}_{2}$ are very close to the VBM.

\section{E. Diffusion of helium in $\mathrm{ThO}_{2}$}

In order to determine the structure influence of helium impurity on $\mathrm{ThO}_{2}$, we systematically calculate the incorporation energy and diffusion energy barriers for helium in both the defect-free and defective $\mathrm{ThO}_{2}$. The incorporation energy is defined to be the energy required to incorporate one helium atom at a pre-existing vacancy or at an interstitial site. In this way, $E_{i n c}$ can be expressed as follows

$$
E_{\text {inc }}=E_{\text {tot }}-E_{\mathrm{ThO}_{2}}-E_{\mathrm{He}}
$$


(a)

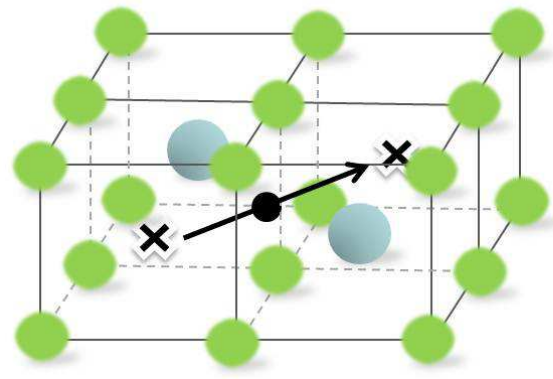

(b)

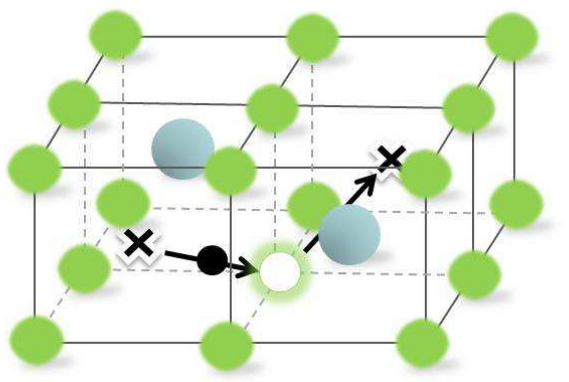

(c)

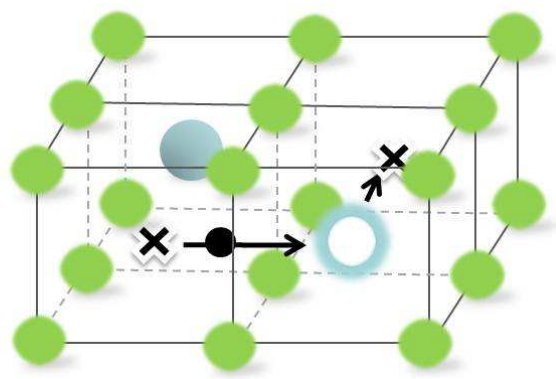
Th $\mathrm{V}_{\mathrm{Th}} \bigcirc \mathrm{V}_{\mathrm{O}} \bigcirc \mathrm{O} \boldsymbol{x}$ OIS $\bullet \mathrm{He}$

FIG. 10: (Color online) Diffusion pathways for a helium atom from one octahedral interstitial site to another octahedral interstitial site directly in intrinsic $\mathrm{ThO}_{2}$ (a), through an oxygen vacancy (b) and through a thorium vacancy in defective $\mathrm{ThO} \mathrm{O}_{2}$ (c).
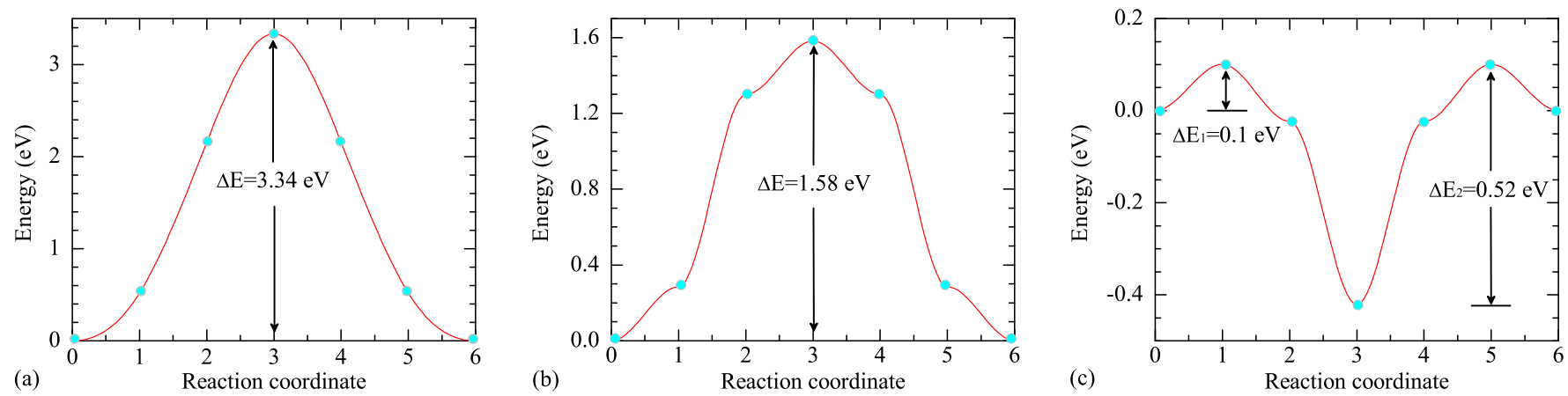

FIG. 11: (Color online) The energy profiles for a helium atom to diffuse from one octahedral interstitial site to another octahedral interstitial site directly in intrinsic $\mathrm{ThO}_{2}$ (a), through an oxygen vacancy (b) and through a thorium vacancy in defective $\mathrm{ThO}_{2}$ (c).

where $E_{\text {tot }}$ is the energy of the $\mathrm{ThO}_{2}$ supercell with an incorporated helium, $E_{\mathrm{ThO}_{2}}$ is the energy of the $\mathrm{ThO}_{2}$ supercell with or without defects, and $E_{\mathrm{He}}$ the energy of an isolated helium atom. Three different kinds of incorporations are systematically considered here, i.e., a helium atom at the oxygen $(\mathrm{O})$ and thorium vacancy $(\mathrm{Th})$ sites in defective $\mathrm{ThO}_{2}$, and a helium atom at the octahedral interstitial site (OIS). The calculated incorporation energies are $2.48,0.21$, and $0.77 \mathrm{eV}$ respectively. We can see that the most possible incorporation site for helium is the thorium vacancy site in defective $\mathrm{ThO}_{2}$. In comparison, the oxygen vacancy site is much less possible for helium incorporation.

The structural influence of helium on $\mathrm{ThO}_{2}$ is determined not only by the incorporation energy, but also by the minimum energy path for helium diffusion. The climbing image nudged elastic band (CINEB) method [56] is then employed to find the minimum-energy diffusion pathways. Based on the obtained incorporation results for helium in $\mathrm{ThO}_{2}$, we here investigate the dif- fusions of helium from OIS to OIS in both defect-free and defective $\mathrm{ThO}_{2}$. Figures 10(a)-(c) depict our considered diffusion ways. And the energy profiles along these paths are shown in Figs. 11(a)-(c) respectively. We can see from Fig. 11(a) that in intrinsic $\mathrm{ThO}_{2}$, the incorporated helium atom needs to overcome a $3.34 \mathrm{eV}$ energy barrier in order to diffuse from one OIS to another. This huge diffusion energy barrier means that at normal temperatures, helium diffusion in intrinsic $\mathrm{ThO}_{2}$ is almost impossible. In the corresponding saddle point along this diffusion path, the helium atom is at the middle of the two OISs, and the nearest oxygen atoms along the [001] direction are repelled from each other by about $0.80 \AA$.

From the calculated energy profile shown in Fig. 11(b), we see that the energy barrier for an incorporated helium atom to diffuse from one OIS to another by passing an oxygen vacancy is lowered down to be $1.58 \mathrm{eV}$. This energy barrier is however still too large for the diffusion to happen at room temperatures. The only possible way for helium to diffuse in $\mathrm{ThO}_{2}$ is found to be passing through 
a thorium vacancy, as depicted in Fig. 10(c). We can see from Fig. 11(c) that the helium atom only needs to overcome a $0.10 \mathrm{eV}$ energy barrier to diffuse from an OIS to the thorium vacancy, and a $0.52 \mathrm{eV}$ energy barrier to diffuse from the thorium vacancy to another OIS. The small energy barriers for helium to diffuse in thorium vacancy-included $\mathrm{ThO}_{2}$ indicate that thorium vacancies might lead to helium aggregation causing failure of the $\mathrm{ThO}_{2}$ material. Fortunately from our above defect formation studies, the formation of thorium vacancies is almost forbidden when the Fermi energy is not upshifted, because of their too large formation energy in $\mathrm{ThO}_{2}$. Therefore, to keep $\mathrm{ThO}_{2}$ away from structural damages from helium incorporation, any kinds of factors possibly leading to upshifts of the Fermi energy should be avoided.

\section{SUMMARY}

In summary, we have performed a systematic firstprinciples study to investigate the thermodynamic properties and structural stabilities of $\mathrm{ThO}_{2}$. Based on the calculated phonon dispersion curves for $\mathrm{ThO}_{2}$, we systematically analyze its thermodynamic properties and obtain the values of its thermal expansion coefficient, bulk modulus, and heat capacities at different temperatures, which are in good agreement with corresponding experimental measurements. The agreement between our calculations and experiments also proves the validity of our methods and model, and the effectiveness of the quasi-harmonic approximation. According to the Umklapp interaction mechanism between different phonon branches, we systematically obtain the mode Grüneisen parameters, and further calculate the thermal conductivities of $\mathrm{ThO}_{2}$. Within the temperature range from Debye temperature to about $1500 \mathrm{~K}$, our calculated thermal conductivity accords very well with experimental results.

In addition to studying the thermodynamic properties of $\mathrm{ThO}_{2}$, we also investigate its structural stability by calculating the formation energy of different defects, and the diffusion behaviors of helium, during which different charge states of the defects are considered. The formation energy results indicate that without any shifts of the Fermi energy, the interstitial $\mathrm{Th}^{4+}$ defect is very probable to appear in $\mathrm{ThO}_{2}$ with an energy release of 1.74 $\mathrm{eV}$. With changing the Fermi energy to different values, the formation possibilities of different defects varies. For helium incorporation, it is found that the helium atom tends to occupy a thorium vacancy in defective $\mathrm{ThO}_{2}$ or occupy the octahedral interstitial site in intrinsic $\mathrm{ThO}_{2}$. It is further revealed that incorporated helium atoms can only diffuse freely in the thorium-vacancy contained $\mathrm{ThO}_{2}$, with small energy barriers of 0.10 and $0.52 \mathrm{eV}$. Our studies point out that to avoid helium damage, the electronic Fermi energy of $\mathrm{ThO}_{2}$ should not be upshifted because it can makes the formation of thorium vacancies less possible.

\section{ACKNOWLEDGMENTS}

This work was supported by NSFC under Grant No. 51071032 , and by Foundations for Development of Science and Technology of China Academy of Engineering Physics under Grants No. 2011A0301016 and No. 2011B0301060.
[1] J. Belle, J. Nucl. Mater. 30, 3 (1969).

[2] J. C. Killeen, J. Nucl. Mater. 88, 185 (1980).

[3] K. N. Kudin, G. E. Scuseria, and R. L. Martin, Phys. Rev. Lett. 89, 266402 (2002).

[4] B. Dorado, B. Amadon, M. Freyss, and M. Bertolus, Phys. Rev. B 79, 235125 (2009).

[5] B. Dorado, G. Jomard, M. Freyss, and M. Bertolus, Phys. Rev. B 82, 035114 (2010).

[6] L. Petit, A. Svane, Z. Szotek, W. M. Temmerman, and G. M. Stocks, Phys. Rev. B 81, 045108 (2010).

[7] J. S. Herring, P. E. MacDonald, K. D. Weaver, C. Kullberg, Nucl. Eng. Des. 203, 65-85 (2001).

[8] J. Tommasi, A. Puill, and Y.K. Lee, "Reactors with Th/Pu Fuels," Proc. Workshop Advanced Reactors with Innovative Fuels, Villigen, Switzerland, October 21-23 (1998).

[9] R. Maitura, Nucl. Rep. 46-53 (2005).

[10] H. Chang, Y. Yang, X. Jing, and Y. Xu, "Thorium-Based Fuel Cycles in the Modular High Temperature Reactor," Tsinghua Sci. Tech. 11, 6 (2006).

[11] A. Carrera, J. Lacouture, C. Campo, and G. Paredes, "Feasibility Study of Boiling Water Reactor Core Based on Thorium-Uranium Fuel Concept," Energy Conversion
Management, 49, 1 (2007).

[12] P. E. Juhn, "Thorium Fuel Cycle Options for Advanced Reactors: Overview of IAEA Activities," Proc. Workshop Advanced Reactors with Innovative Fuels, Villigen, Switzerland, October 21-23 (1998).

[13] A. C. Momin and Karkhanvala, High Temp. Sci. 10, 45 (1978).

[14] J. R. Springer, E. A. Eldridge, M. U. Goodyear, T. R. Wright and J. F. Lagedrast Report No. BMI-X-10210 (1968).

[15] J. C. Weilbacher, High Temp.-High Press. 4, 431 (1972).

[16] D. L. McElroy, J. P. Moore, P. H. Spindler, Oak Ridge National Laboratory Report ORNL-4429, p. 121 (1968).

[17] Armour Research Foundation ARF-Project No. 6-025, Final Report, (1957).

[18] M. Murabayashi, J. Nucl. Sci. Technol. 7, 559 (1970).

[19] C. G. S. Pillai, P. Raj, J. Nucl. Mater. 277, 116-119 (2000)

[20] W. G. Bradshaw, C. O. Mathews, Report LMSD-2466, (1958).

[21] J. P. Moore, R. S. Graves, T. G. Kollie, D. L. McEIroy, Oak Ridge National Laboratory Report ORNL-4121, (1967). 
[22] P. Srirama Mufti, C. K. Mathews, J. Phys. D 24, 2202 (1991).

[23] K. Bakker, E. H. P. Corfunke, R. J. M. Konings and R. P. C. Scharm, J. Nucl. Mater. 250, 1-12 (1997).

[24] D. Chadwick and J. Graham, Nat. Phys. Sci. 237, 127 (1972).

[25] G. C. Allen and P. M. Tucker, J. Chem. Soc. Dalton p. 470 (1973).

[26] B. W. Veal and D. J. Lam, Phys. Rev. B 10, 12 (1974).

[27] A. Jayaraman, G. A. Kourouklis, L. G. Van Uitert, Pramana 30, 225 (1988).

[28] J. P. Dancausse, E. Gering, S. Heathman, U. Benedict, High Pressure Res. 2, 381 (1990).

[29] M. Idiri, T. Le Bihan, S. Heathman, J. Rebizant, Phys. Rev. B 70, 014113 (2004).

[30] Y. -E. Kim, J. -W. Park and J. Cleveland, "Thermophysical properties database of materials for light water reactors and heavy water reactors", Vienna, (2006).

[31] C. Sevik and T. Cagin, Phys. Rev. B 80, 014108 (2009).

[32] V. Kanchana, G. Vaitheeswaran, A. Svane, A. Delin, J. Phys.: Condens. Matter 18, 9615 (2006).

[33] I. R. Shein, K. I. Shein, A. L. Ivanovskii, J. Nucl. Mater. 361, 69 (2007).

[34] J. C. Boettger, Int. J. Quantum. Chem. 109, 3564 (2009).

[35] B. T. Wang, H. L. Shi , W. D. Li , P. Zhang, J. Nucl. Mater. 399, 181-188 (2010).

[36] A. Boudjemline, L. Louail, Mazharul M. Islam, B. Diawara, Comp. Mater. Sci. 50, 2280-2286 (2011).

[37] G. Kresse, J. Furthmüller, computer code VASP, Vienna, (2005).

[38] G. Kresse, J. Furthmüller, Phys. Rev. B 54, 11169 (1996).
[39] P. E. Blöchl, Phys. Rev. B 50, 17953 (1994).

[40] J. P. Perdew, K. Burke, M. Ernzerhof, Phys. Rev. Lett. 77, 3865 (1996).

[41] H. J. Monkhorst, J. D. Pack, Phys. Rev. B 13, 5188 (1976).

[42] A. Baldereschi, S. Baroni, R. Resta, Phys. Rev. Lett. 61, 734 (1988).

[43] M. Peressi, N. Binggeli, A. Baldereschi, J. Phys. D 31, 1273 (1998).

[44] F. Brich, Phys. Rev. 71, 809 (1947).

[45] J. S. Olsen, L. Gerward, V. Kanchana, G. Vaitheeswaran, J. Alloys Compd. 381, 37 (2004).

[46] Y. Lu, D. F. Li, R. W. Li, H. L. Shi, P. Zhang, J. Nucl. Mater. 408, 136-141 (2011).

[47] P. Zhang, B. T. Wang, and X. G. Zhao, Phys. Rev. B 82, $144110(2010)$.

[48] P. M. Macedo, W. Capps, J. B. Watchman, J. Am. Ceram. Soc. 47, 651 (1964).

[49] Y. S. Touloukian, R. K. Kirby, R. E. Taylor, T. Y. R. Lee, Thermal Expansion. Nonmetallic Solids (IFI/Plenum, New York, 1970).

[50] G. A. Slack, J. Phys. Chem. Solids 34, 321 (1973).

[51] G. A. Slack, Solid State Phys. 34, 1 (1979).

[52] O. L. Anderson, J. Phys. Chem. Solids 12, 41 (1959).

[53] A. A. Blanco, E. Francisco, and V. Luana, Comput. Phys. Commun. 158, 57 (2004).

[54] E. Francisco, J. M. Recio, M. A. Blanco, A. Martín Pendás, J. Phys. Chem. 102, 1595 (1998).

[55] E. Francisco, M. A. Blanco, and G. Sanjurjo, Phys. Rev. B 63, 094107 (2001).

[56] G. Henkelman, B. P. Uberuaga, and H. Jonsson, J. Chem. Phys. 113, 9901 (2000). 